\title{
Research on Cascading Failure Model of Urban Regional Traffic Network under Random Attacks
}

\author{
Ruru Xing $\mathbb{D}^{1}{ }^{1}$ Qingfang Yang, ${ }^{1,2}$ and Lili Zheng $\mathbb{D}^{1,2}$ \\ ${ }^{1}$ College of Transportation, Jilin University, Changchun 130022, China \\ ${ }^{2}$ State Key Laboratory of Automobile Simulation and Control, Jilin University, Changchun 130022, China \\ Correspondence should be addressed to Lili Zheng; lilizheng@jlu.edu.cn
}

Received 18 August 2018; Revised 5 October 2018; Accepted 25 October 2018; Published 11 November 2018

Academic Editor: Florentino Borondo

Copyright (c) 2018 Ruru Xing et al. This is an open access article distributed under the Creative Commons Attribution License, which permits unrestricted use, distribution, and reproduction in any medium, provided the original work is properly cited.

For better describing the network cascading failure caused by random attacks such as traffic accidents, this paper introduces the ORNL-PSerc-Alaska (OPA) model to analyze the urban regional traffic network vulnerability. Firstly, the double-layer network model is built for analyzing the complex characteristics of the regional traffic network. Secondly, the cascading failure model is built to better describe the process of the network cascading failure under random attacks. Finally, by simulating the network failure process, the vulnerability of the traffic network will be pointed out, which provides the theoretical basis for avoiding the network cascading failure under random attacks.

\section{Introduction}

Real networks often suffer from various external attacks at any time, which triggers self-organizing reactions within the networks. The antidestruction performance of the network directly affects the recovery function of the network after an attack. If the network cannot defend against the attack, a vicious cycle will occur. For example, a heavy rain occurred in Jinan on July 18, 2007, which is different from the torrential rains in the past. This convective weather leads to huge destruction of the urban drainage system. It showed waterlogging and the traffic network got failed in an instant. At the same time, it had caused many personal injuries and serious economic losses. For a time, various service functions of the urban network were difficult to restore. This unexpected incident has caused the attention of the Jinan municipal government and has also triggered researchers to think deeply about the vulnerability of the urban traffic network. In fact, there is a phenomenon of the network vulnerability hidden behind this incident; Cascading Failure.

Based on $[1,2]$, this paper firstly proposes the definition of cascading failure of the urban traffic network. In the urban traffic network, if one or more intersections (or roads) are congested, other vehicles will adjust travel paths in time to bypass the congested intersections (or roads). Because of the traffic flow redistribution, some neighboring intersections (or roads) will be oversaturated, which generates the chain effects and leads to the collapse of the entire urban regional traffic network.

Complex network cascading failure model includes loadcapacity model [3-6], binary influence model [7], sandpile model [7, 8], ORNL-PSerc-Alaska (OPA) model [9], CASCADE model [10], coupled map lattice model [11], and disaster spread dynamic model $[12,13]$.

Recently, the research on the cascading failure model of the urban traffic network mainly focuses on the loadcapacity model, which assumes that the capacity is directly proportional to the initial load [14-16] and simulates the evolution process of the network cascading failure under different attack modes [14, 17]. Zheng et al. [16] proposed a Crucitti-Latora-Marchiori (CLM) optimization model based on the characteristics of the urban traffic networks, introduced congestion effects to determine node loads, and introduced actual capacity into the cost function. The results showed that the optimization model reflects the urban traffic network cascading failure. Wang et al. [18] proposed a dynamic model of disaster contagion and simulated the evolution of the urban traffic network cascading failure. Peng [19] established a dynamic 
model of disaster propagation based on the double-layer network model of the urban traffic network. After the simulation and analysis of self-repair factors and other parameters, the model can simulate the evolution process of the network failure.

Based on the above analysis, the existing research mostly focuses on how the cascading failure model describes the urban failure process by considering the selection of model parameters. Recently, the causes of the urban regional network cascading failure are divided into two types: (1) random attacks; (2) deliberate attacks. Reference [3] pointed out that the actual network can resist the failure caused by random attacks. Deliberately attacking the network can easily cause the network cascading failure and bring about a wide range of damage. However, in the actual urban traffic network, random attacks such as traffic accidents have a greater impact on the traffic network. Therefore, the research on urban cascading failure problem needs to consider the attack mode. This article focuses on building a cascading failure model that can describe the urban cascading failure phenomenon under random attacks.

At present, there are several studies on cascading failure under random attacks. Cheng et al. [20] studied the robustness of interdependent Erdös-Rényi (ER) random networks under random attacks and further analyzed the network robustness under different average node degrees. Huang et al. [21] used percolation theory to study the robustness of the cascading failure in cyber physical systems under random attacks. The simulation results showed that the network size has no effect on the system robustness. Li et al. [22] proposed the cascading failure model in scalefree (SF) networks, Watts-Strogatz (WS) networks, and ER networks by considering random attacks and analyzed the cascading failure process of three networks compared with the highest-load attack. Chattopadhyay et al. [23] provided the optimal interdependence structures in order to improve the network robustness under random attacks. Ruj et al. [24] modeled the network cascading failure of smart grids under random attacks and deliberate attacks, which will increase the resilience of the smart grid. Shen et al. [25] proposed a load-capacity model to study the interdependent network cascading failure and found the failure law of the interdependent network under random attacks.

In the previous studies aimed at the cascading failure models, some of them focused on the factors that affect the network robustness, while others focused on building models of improving the network ability of resisting random attacks. In this paper, the vulnerability of the traffic network is analyzed in depth by establishing a cascade failure model under random attacks. Therefore, this paper will mainly be reflected in how to excavate the vulnerable position of the traffic network which is easy to cause the cascade failure in the face of random attacks.

For better describing the network cascading failure caused by random attacks such as the traffic accidents, this paper introduces the OPA model to analyze the regional traffic network vulnerability. During the simulation process, the vulnerable position of the traffic network can be obtained. The outstanding contributions of this article are as follows:
(1) The OPA model as a grid cascading failure model is first introduced to research the cascading failure of the urban traffic network under random attacks.

(2) In order to accurately describe the essence of the process of the traffic network cascading failure (that is, the temporary loss of traffic capacity), this paper redefines the update rule of traffic impedance after the road getting failed and the traffic impedance of the failed road will be adjusted to infinity, which is different from the method of directly deleting the failed road in some current studies. The method of this paper influences the choice of travel path by the dynamic change of road traffic impedance, instead of artificially changing the network topology.

(3) Different from the method of constructing a scalefree network for failure simulation in most studies, this paper selects the actual traffic data of a regional urban traffic network as the cascade failure simulation object, which can better reflect the law of the traffic network cascade failure.

This article is organized as follows. Section 2 first builds a double-layer urban regional traffic network model to provide a basis for analyzing the road network failure dynamic characteristics, and then establishes a cascade failure model to reflect the urban regional traffic network failure process under random attacks. Section 3 confirms the feasibility of the model and analyzes the vulnerability of the urban regional traffic network by simulating the cascade failure process. In Section 4, conclusions and directions for future research are presented.

\section{Modeling on Urban Regional Network Cascading Failure}

The research shows that the network topology directly affects the layout of the network function. Moreover, the network topology also determines the characteristics of the network itself, including the static statistical characteristics and the dynamics behavior [1]. In other words, the occurrence of cascading failure not only depends on the operational state of traffic flow, but also on the irrational design of the infrastructure. Therefore, how to analyze the urban regional traffic network cascade failure from the two perspectives of dynamics behavior and network topology is the first research focus of this part. The urban regional traffic network is composed of a mixture of regional road network and regional travel network. In order to solve the above problem, Section 2.1 establishes a double-layer network model of urban regional road network from the two aspects of the road network and travel network, which can solve the above problem.

In addition, Porta et al. [26] analyzed the network topology of six urban traffic networks and found that their networks are scale-free networks. For the performance of a scale-free network under attacks, [27-29] pointed out that if $80 \%$ of nodes are failed by random attacks, the remaining network will be likely to form a complete community and maintain connectivity. However, if $5 \%-10 \%$ of important nodes get failed at the same time, it will cause the network to collapse to a small group. Researchers such as Albert et al. 


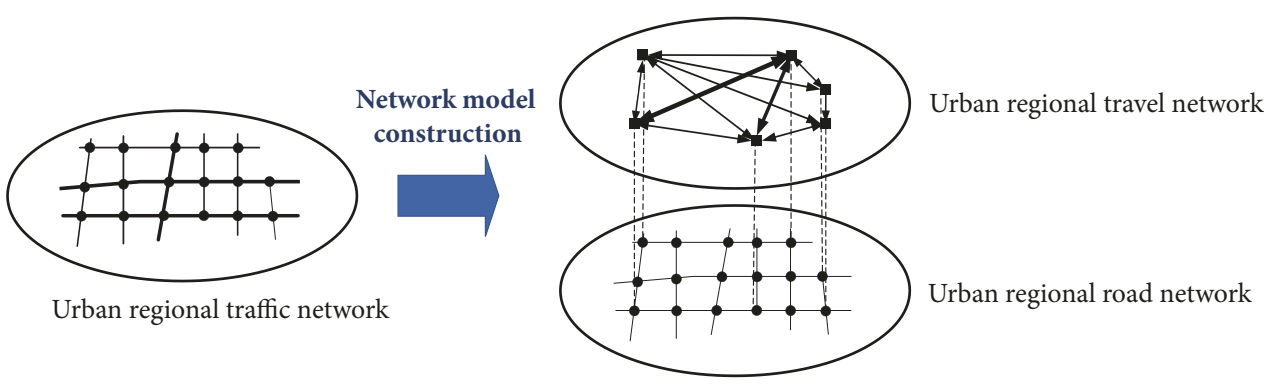

FIGURE 1: Double-layer network model of the urban regional traffic network.

have verified that the scale-free network satisfies the above law through a large number of experiments. Then, the urban traffic network not only meets the characteristics of scale-free networks, but also has the features of small-world networks [26]. In the face of random attacks, which kind of road getting failed can lead to the huge damage of the traffic network. For this, Section 2.2 establishes the cascade failure model under random attacks to lay the foundation for analyzing the vulnerability of the regional traffic network.

2.1. Double-Layer Network Model. Reference [1] showed clearly that the urban traffic network topology and its function features are inseparable and affect each other. Therefore, it is unreasonable to research the dynamic characteristics in urban regional traffic network by using the network topology structure. But, the urban traffic network dynamic characteristic can be researched by combining with the traffic flow travel network. From the above, the research on Section 2.1 divides the regional traffic network into two parts, regional road network and regional travel network, and builds a doublelayer network model of the urban regional traffic network in Figure 1.

In Figure 1, the black dots represent the intersections in the urban regional traffic network and the road network. The lines represent the roads and the width of the lines represents the traffic volume of the roads in the traffic network. In the travel network, the black blocks represent the OriginDestination (OD) points of traffic flow and the arrows of the line reflect the directions of traffic flow.

Besides, the specific meaning of this model is divided in two aspects:

(1) The lower network represents the topology map of the urban regional road network $G_{A}=(\mathscr{V}, \mathscr{K}, \mathscr{W})$, where $\mathscr{V}$ represents the node set (namely, a collection of intersections in the urban regional traffic network), $\mathscr{K}$ represents the edge set (namely, a collection of roads connected between intersections), and $\mathscr{W}$ represents the weight set (namely, a collection of road impedances).

(2) The upper network represents the urban regional travel network $G_{B}=(\mathscr{P}, \mathscr{Z}, \mathscr{Q})$, where $\mathscr{P}$ represents OD point set, $\mathscr{Z}$ represents the set of the relationships between OD points (if travel point $i$ and travel point $j$ are connected, it means they are associated), and $Q$ represents the traffic flow set (namely, traffic volume between each OD point).
The lower road network and the upper travel network affect each other:

(1) The upper travel network determines the traffic volume during the traffic distribution. The values of traffic volume change the values of the traffic impedance in the lower road network, namely, $\forall q_{i j} \in \mathbb{Q}$ and $\exists w_{i j} \in \mathscr{W}, q_{i j} \longrightarrow w_{i j}$.

(2) The changes of the traffic impedance in the lower road network also change the traffic volume in the upper travel network, namely, $\forall w_{i j}^{\prime} \in \mathscr{W}$ and $\exists q_{i j}^{\prime} \in \mathbb{Q}, w_{i j}^{\prime} \longrightarrow q_{i j}^{\prime}$.

(3) The values of traffic volume in turn affect the choices of the travel route. What is more, the changes of the travel route will affect the redistribution of traffic flow within the travel network.

\subsection{Cascading Failure Model Based on OPA}

\subsubsection{Model Parameter}

(1) Load. The load is a related parameter in a complex network, and can be defined by matter, information, or energy. Therefore, the load can be defined as a specific or abstract parameter [3]. Reference [30] indicated that the node load of one general network is determined by the degree or the betweenness of the nodes. However, these two kinds of statistical parameters are determined by the network topology and cannot directly reflect the dynamic characteristics of the network. Therefore, the node load is defined as the traffic volume in the upper travel network:

$$
l_{i}(t)=q_{i}(t)
$$

where $l_{i}(t)$ represents the load of node $i$ at $t$ time; $q_{i}(t)$ represents the traffic volume of intersection $i$ at $t$ time in the upper travel network.

Similarly, referring to the definition of the node load, the definition of the edge load can also be obtained.

$$
l_{i j}(t)=q_{i j}(t)
$$

where $l_{i j}(t)$ represents the load of edge $e_{i j}$ at $t$ time; $q_{i j}(t)$ represents the traffic volume of road $e_{i j}$ at $t$ time in the upper travel network.

(2) Capacity. According to the cascading failure theory, the node bearing capacity (or capacity) refers to the maximum load which a node can bear [31]. From (1), we can see that 
the load of a node is defined as a continuous function that changes dynamically with time. Especially when the urban regional traffic network is attacked, the node load directly affects the traffic flow distribution of the upper travel network and the load will be redistributed. If the redistributed node load exceeds the maximum load which it cannot tolerate, the node will lose its functionality after the attacks and the failure of this node can easily trigger the cascading failure. Hence, the bearing capacity can also be understood as the critical threshold of a failed node (or edge).

In the urban regional traffic network, the traffic volume should not be too large to cause the traffic congestion. When the traffic volume is too large to exceed the maximum bearing capacity, the road will lose the transportation service due to the traffic congestion. Therefore, the traffic volume is within the range of free-flow volume to the maximum load. The node load should be maintained within the specified maximum flow $q^{\max }$. Based on the above, the load capacity $C_{i}$ of node $i$ in the travel network is defined as its maximum flow $q_{i}^{\max }$.

$$
C_{i}=q_{i}^{\max }
$$

where $q_{i}^{\max }$ represents the maximum traffic volume of intersection $i$.

Different from the bearing capacity of the intersection in the travel network, the bearing capacity of the road is a function related to its traffic capacity. Reference [17] pointed out that when the traffic volume exceeds 1.15 times of its traffic capacity, the road is failed. Therefore the specific equation for the bearing capacity $C_{i j}$ of road $e_{i j}$ in the travel network is given:

$$
C_{i j}=1.15 c_{i j}
$$

where $c_{i j}$ represents the traffic capacity of road $e_{i j}$ in the upper travel network.

(3) Traffic Impedance. As a parameter that directly affects the travel cost of travelers, traffic impedance has narrow and broad senses. In the narrow sense, the traffic impedance on a road represents the average of the travel time spent by all travelers. The traffic impedance in the broad sense is based on the consideration of the influence of the three elements of people, vehicles, and roads. Reference [32] gave the definition of traffic impedance: the traffic impedance can represent the distance, the travel time, the travel cost and the travel comfort, or a combination of these factors on the traffic network.

The traffic impedance can be applied to different urban traffic networks and be defined in a variety of ways. However, the traffic impedance is composed of two parts, road impedance (edge impedance) and intersection impedance (node impedance).

(1) Road Impedance. In the calculation of travel cost, as the primary consideration, the travel time is particularly important. Most travelers choose the path with the shortest time as their travel route. Therefore, some studies chose the travel time parameter as the road impedance. However, the travel time of one road is a dynamic variable which is closely related to the traffic volume in the traffic network. It can be further understood that the travel time is a function related to the traffic volume.

The Bureau of Public Road (BPR) function proposed by the US Highway Authority is often used as the equation for calculating the road impedance. Therefore, the BPR function is introduced as the calculation equation of the road impedance.

$$
\widehat{t}_{i j}=t_{i j}^{0}\left(1+\alpha\left(\frac{q_{i j}}{c_{i j}}\right)^{\beta}\right)
$$

where $t_{i j}^{0}$ represents the travel time in the free-flow state of traffic flow on the road $e_{i j} ; c_{i j}$ represents the actual traffic capacity of the road $e_{i j} ; q_{i j}$ represents the traffic volume of the road $e_{i j} ; \alpha$ and $\beta$ represent the model parameters, generally $\alpha=0.15$ and $\beta=4$.

(2) Intersection Impedance. The intersection impedance refers to the time spent by the travelers traveling through the intersection. Generally, it can be represented by the intersection delay parameter. Due to the different forms of the urban traffic networks, the forms of intersections are also varied and the calculation equations for the intersection delay are not the same. Webster's method is usually used to calculate the delay $d_{i}$ of the timing signal control intersection:

$$
\begin{aligned}
d_{i}= & \frac{c_{i}\left(1-\lambda_{i}\right)^{2}}{2\left(1-\lambda_{i} x_{i}\right)}+\frac{x_{i}^{2}}{2 q_{i}\left(1-x_{i}\right)} \\
& -0.65\left(\frac{c_{i}}{q_{i}^{2}}\right)^{1 / 3} x_{i}^{\left(2+5 \lambda_{i}\right)}
\end{aligned}
$$

where $c_{i}$ represents the period of the intersection $i$; $q_{i}$ represents the traffic volume at the intersection $i ; x_{i}$ represents the saturation degree of the intersection $i ; \lambda_{i}$ represents the green ratio of the intersection $i$.

(4) Network Failure Evaluation Index. In simulating the cascading failure of the regional traffic network, how to reflect the changes of the network performance and to determine the degree of the network damage become the study of this part. The following parameter variables are introduced to quantitatively evaluate and measure the network damage under the numerical simulation of cascading failure.

(1) Maximum Connected Area. The maximum connected area specifically refers to the number of nodes in the maximum connected area of the network after the attacks. It can reflect the degree of the network connectivity. Therefore, the maximum connected area parameter is able to measure the connectivity of the traffic network under random attacks. The specific equation is as follows [27]:

$$
G=\frac{N^{\prime}}{N}
$$

where $N^{\prime}$ represents the number of nodes in the maximum connected area after the random attacks; $N$ represents the number of nodes in the network. 
From (7), it can be concluded that when $G \approx 1$, there is no failed node after the attack, that is, the traffic network is intact and has good connectivity; when $G \approx 0$, all the nodes in the traffic network are found to be ineffective after the attack, that is, the traffic network is completely collapsed and has no connectivity path.

(2) Global Efficiency. Reference [33] pointed out that the efficiency $\varepsilon_{i j}$ of edge $e_{i j}$ refers to the ability of transmitting a data packet from node $i$ to node $j$. In other words, the global efficiency parameter can reflect the operate ability of traffic flow in the network after facing external attacks, namely, the operation status of the regional traffic network. The specific equation of global efficiency $E_{\text {glob }}$ is as follows:

$$
E_{\text {glob }}=\frac{1}{N(N-1)} \sum_{i, j \in \mathscr{V}, i \neq j} \varepsilon_{i j}=\frac{1}{N(N-1)} \sum_{i, j \in \mathscr{V}, i \neq j} \frac{1}{d_{i j}}
$$

where $N$ represents the number of intersections in the network; $d_{i j}$ represents the number of roads corresponding to the shortest path between intersection $i$ and intersection $j ; \varepsilon_{i j}$ represents the efficiency between intersection $i$ and intersection $j$.

2.2.2. Cascading Failure Model. The OPA model fully explains the cascading failure mechanism of the power grid from the two possible failures [9]. It is usually used to analyze the cascading failure caused by the random disconnection of power grid lines due to the random factors such as weather factor. In other words, the OPA model is able to simulate the power grid cascading failure under random attacks. Therefore, the OPA model is introduced to study the traffic network cascading failure caused by random attacks.

The OPA model divides the grid cascading failure process into slow dynamic processes and fast dynamic processes. Reference [2] put forward that the cascading failure process of the urban traffic network also includes slow failure and fast failure processes, which is the similar with the failure process reflected by the OPA model. Therefore, according to the two process of the OPA model, the process of the traffic network cascading failure model based on OPA is proposed in Figure 2.

(1) Slow Dynamic Process. It is assumed that there are $m$ roads and $n$ intersections in the urban regional traffic. Among these intersections, part of them are the origin points in the travel network, and part of them are the destination points. Then, $O$ represents the set of the traffic volume of the origin intersections, and $D$ represents the set of the traffic volume of the destination intersections. $P_{i k}$ indicates the traffic volume of the intersection $i$ at $k$ time. And $P_{k}$ represents the vector of the traffic volume of all the intersections at $k$ time, namely, $P_{k}=\left(P_{1 k}, P_{2 k}, \ldots, P_{n k}\right)^{T}$. Then, suppose the value of the traffic volume flowing into the intersection $i$ is positive and the value of the traffic volume flowing out the intersection $i$ is negative, and $\sum_{i} P_{i k}=0 . F_{j k}$ indicates the traffic volume on the road $j$ at $k$ time, and $F_{k}=\left(F_{1 k}, F_{2 k}, \ldots, F_{m k}\right)^{T}$ indicates the traffic

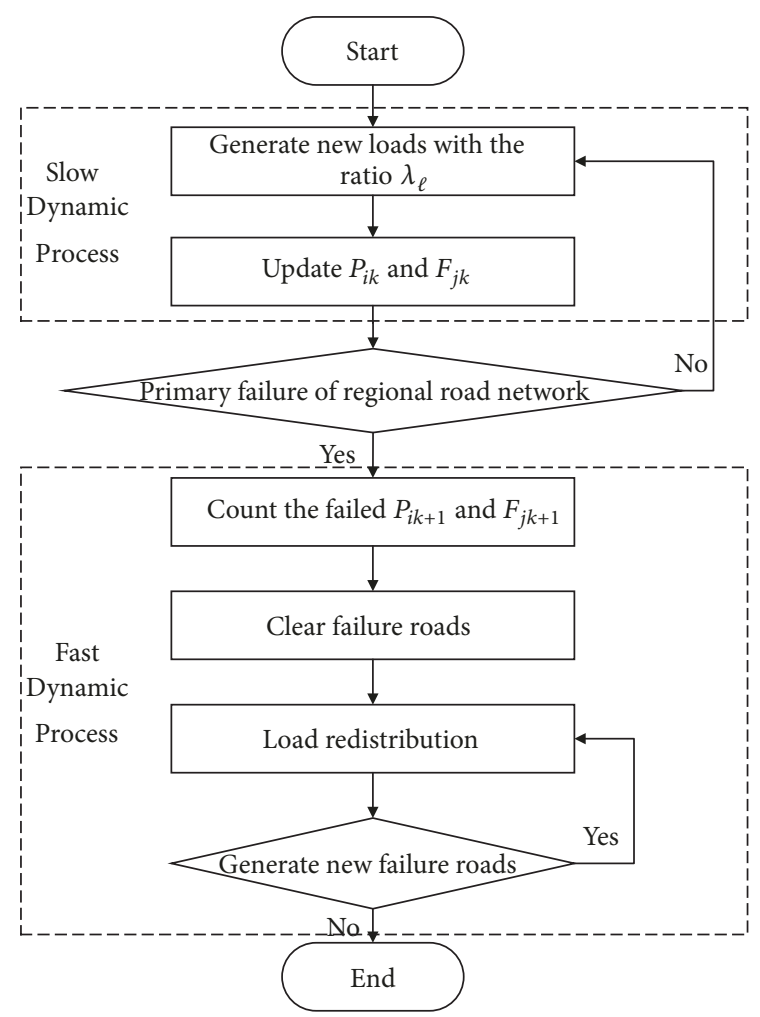

FIGURE 2: Process of the cascading failure model based on OPA.

volume of all roads at $k$ time. In the slow dynamic process, the roads have not reached the failure state in the network.

$$
F_{j k} \leq c_{j}, \quad j=1,2, \ldots, m
$$

where $c_{j}$ represents the traffic capacity of road $j$.

In the urban regional traffic network, the traffic volume on the road is generated by the OD points in the travel network. So, $P_{k}=F_{k}$.

If the ratio of the total traffic volume at $\ell$ time and $\ell+1$ time is $\lambda_{\ell}$, then

$$
P_{k}=P_{0} \prod_{\ell=1}^{k} \lambda_{\ell}
$$

where $P_{0}$ represents the total traffic volume of all intersections at the initial moment.

Then

$$
F_{k}=P_{k}=P_{0} \prod_{\ell=1}^{k} \lambda_{\ell}=F_{0} \prod_{\ell=1}^{k} \lambda_{\ell}
$$

(2) Fast Dynamic Process. There are also two types of random attacks that the urban regional traffic networks faced with.

(1) No Overload Failure. For example, the urban regional traffic network makes the cascading failure caused by severe weather such as heavy rain and heavy snow. Under normal 


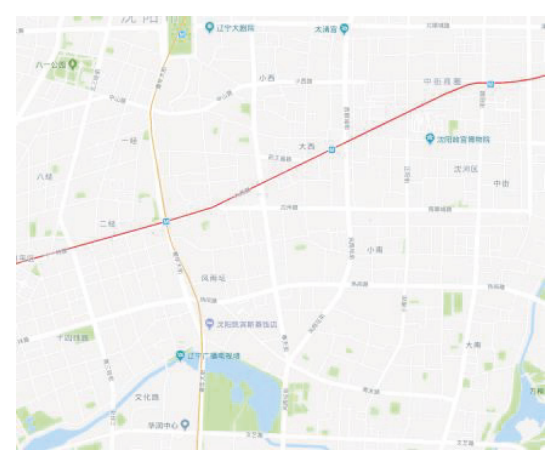

(a)

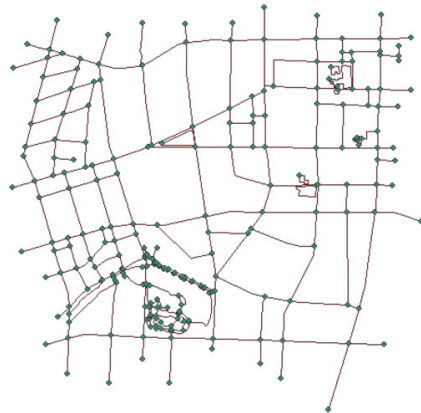

(b)

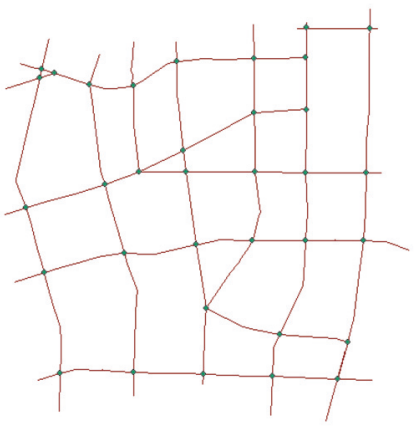

(c)

FIGURE 3: Urban complex network model building process: (a) real regional traffic network; (b) network abstract topology; (c) regional network model.

circumstances, the occurrence probability of this type of failures is related to the occurrence probability of bad weather. And it can be understood as a probability that the value is small, that is,

$$
P\{\operatorname{road} j \text { is failed }\}=h_{0}\left(M_{j k}\right)
$$

where $h_{0}$ is a positive nondecreasing function.

(2) Overload Failure. For example, a traffic accident causes the traffic congestion in traffic network. The traffic volume of the road exceeds its bearing capacity, which leads to the traffic network cascading failure. This overload failure has a high value of the occurrence probability on accident-prone roads, namely

$$
P\{\operatorname{road} j \text { is failed }\}=h_{1}\left(M_{j k}\right)
$$

where $h_{1}$ is a positive nondecreasing function and $h_{1} \gg h_{0}$.

Assuming that the traffic network cascading failure occurs at $k$ time, the traffic volume on the road $j$ at $k$ time is defined as $f_{j}$, and $f=\left(f_{1}, f_{2}, \ldots, f_{m}\right)^{T}$ represents the vector of the traffic volume on all the roads at $k$ time. If $p_{i}$ represents the traffic volume of the intersection $i$ at $k$ time, $p=\left(p_{1}, p_{2}, \ldots, p_{n}\right)^{T}$ indicates the vector of the traffic volume of all the intersections at $k$ time. Then, $f$ and $p$ are initialized to

$$
\begin{aligned}
& f=F_{k} \\
& p=P_{k}
\end{aligned}
$$

(3) Failure Propagation Algorithm. The process of the traffic network failure propagation algorithm based on OPA is as follows:

Step 1 (initialization). According to (14) and (15), the traffic volume of each intersection and road is initialized after the failure occurred.

Step 2 (identify the invalidation section). According to (12) and (13), the failed road in the traffic network is determined.
Step 3 (load redistribution). According to the UE balanced allocation, the loads in the traffic network are redistributed.

Step 4 (failure judgment). According to (12) and (13), if there is a new failed road, continue to Step 3; otherwise go to Step 5 and end the process.

Step 5. End.

\section{Case Study}

3.1. Complex Analysis of Urban Regional Traffic Network. This section selects a regional network data collected from Shenyang, China. Use ArcGIS to process spatial data in Figure 3. The intersections and roads of this regional network are represented by nodes and edges respectively. By combining the Primal approach, it realizes the transformation from Figures 3(b) and 3(c), which obtains the complex network model of the regional network in Shenyang.

The cascading failure theory is part of complex theory. For applying the cascading failure theory, it needs to verify that the regional traffic network has complex characteristics. In other words, the topology parameters of the traffic network meet the characteristics of complex network. Therefore, Sections 3.1.1 and 3.1.2 analyze the complex characteristics of the regional traffic network.

3.1.1. Network Eigenvalue Calculation. The most important two criteria of judging whether a real network is complex networks are (1) small-world effects; (2) scale-free degree distribution [34]. In addition, the two criteria are described by some important statistical features of complex networks. Therefore, the average node degrees $\langle k\rangle$, the average shortest path length $L$, and the average clustering coefficient $C$ are introduced to describe the complex characteristic of the urban regional traffic.

The degree $k_{i}$ of node $i$ represents the number of adjacent edges of the node $i$. The network average node degree $\langle k\rangle$ 
TABLE 1: Shenyang regional traffic network characteristics.

\begin{tabular}{lllccccc}
\hline Network feature value & $N$ & $E$ & $\langle k\rangle$ & $L$ & $C$ & $L_{R}$ \\
\hline Calculation results & 34 & 55 & 3.8824 & 5.7971 & 0.4180 & 5.1231 & 0.0837 \\
\hline
\end{tabular}

refers to the average of the degrees $k_{i}$ of all nodes in the network.

$$
\langle k\rangle=\frac{1}{N} \sum_{i=1}^{N} k_{i}
$$

The average distance between nodes in the network is defined as the average shortest path length of the network $L$. The average shortest path length $L$ in the network specifically describes the average degree of separation between pairs of nodes, which reflects the size of the network. The calculation formula is

$$
L=\frac{1}{N(N-1)} \sum_{i, j \in \mathscr{V}, i \neq j} d_{i j}
$$

The network average clustering coefficient $C$ is the average of the clustering coefficients $C_{i}$ of all nodes.

$$
C=\frac{1}{N} \sum_{i} C_{i}
$$

And, the clustering coefficient $C_{i}$ of node $i$ is defined as

$$
C_{i}=\frac{2 M_{i}}{k_{i}\left(k_{i}-1\right)}
$$

where $k_{i}$ represents the number of nodes connected to the node $i$ and $M_{i}$ represents the number of edges connected between $k_{i}$ nodes.

By calculating the regional network topological adjacency matrix and the distance of adjacency matrix at the same time, the number of nodes $N$, the number of edges $E$, the average node degrees $\langle k\rangle$, the average shortest path length $L$, and the average clustering coefficient $C$ can be obtained. And, compare the average shortest path length $L_{R}$ and the average clustering coefficient $C_{R}$ of random network. The calculation result is as shown in Table 1.

It can be obtained from Table 1 :

(1) Shenyang regional traffic network includes 34 intersections and 55 roads. The average node degree is about 3.89 , which is between 3 and $4 .\langle k\rangle \approx 4$ shows that most intersection in the regional traffic network is connected to 4 roads.

(2) Reference [35] pointed that when the average shortest path length of the network $L$ is almost as small as the one of random network $L_{R}$ and the average clustering coefficient of the network $C$ is far larger than the one of random network $C_{R}$, the network has the small-world characteristics. And, it can be seen that the regional traffic network has small-world network characteristics in Table 1, namely, $L$ is almost as small as $L_{R}$ and $C \gg C_{R}$.
TABLE 2: Statistics of node degree in Shenyang regional traffic network.

\begin{tabular}{lcc}
\hline Node degree & 3 & 4 \\
\hline The number of nodes & 4 & 30 \\
Proportion (\%) & 11.76 & 88.24 \\
\hline
\end{tabular}

3.1.2. Network Eigenvalue Analysis. Firstly, the distribution of node degrees is analyzed to grasp the specific spatial distribution of nodes in the urban regional traffic network. The statistical analysis of all the node degrees shows in Table 2.

As can be seen from Table 2, the proportion of nodes with the node degree of 3 is $11.76 \%$ and the proportion of nodes with the node degree of 4 is $88.24 \%$. In addition, the number of nodes with the node degree of 4 is the highest, which also directly reflects that the intersections in this regional traffic network are often connected with 4 roads.

Urban traffic network can be abstracted to a weighted complex network. Different from simple weighted complex network, urban road traffic network is a network with complex topological structure composed of different level roads and different size intersections. The common intersections in the urban traffic network are roughly five-way intersections, four-way intersections and three-way intersections, where four-way intersection is the most widely used. According to the definition of the node degree in the directed graph, all four intersections in the network have the same in-degree $k_{i}^{\text {in }}$ and out-degree $k_{i}^{\text {out }}$, and the values are 4 , namely, $k_{i}^{\text {in }}=$ $k_{i}^{\text {out }}=4$. However, as the four-way intersections, the size and the capacity of every intersection are different. The above node degree calculation is based on the complex network definition. Hence, the new definitions of the in-degree $k_{i}^{i n}$, out-degree $k_{i}^{\text {out }}$, and node degree $k_{i}$ of urban road traffic network are given.

$$
\begin{aligned}
k_{i}^{i n} & =\sum_{j \in \mathscr{V}} a_{j i}^{\prime} \\
k_{i}^{\text {out }} & =\sum_{j \in \mathscr{V}} a_{i j}^{\prime} \\
k_{i} & =k_{i}^{\text {in }}+k_{i}^{\text {out }}
\end{aligned}
$$

where $a_{i j}^{\prime}$ represents the number of lanes from the intersection $i$ to the intersection $j ; d_{i j}$ represents the number of lanes from the intersection $j$ to the intersection $i$.

Based on Table 3, the statistical results of the node degree of the traffic network are shown in Figure 4 by using MATLAB.

Figure 4 is a regression analysis of the cumulative distribution of node degrees from the perspective of the power law and linear distributions. In Figure 4(a), $P_{k} \propto$ 


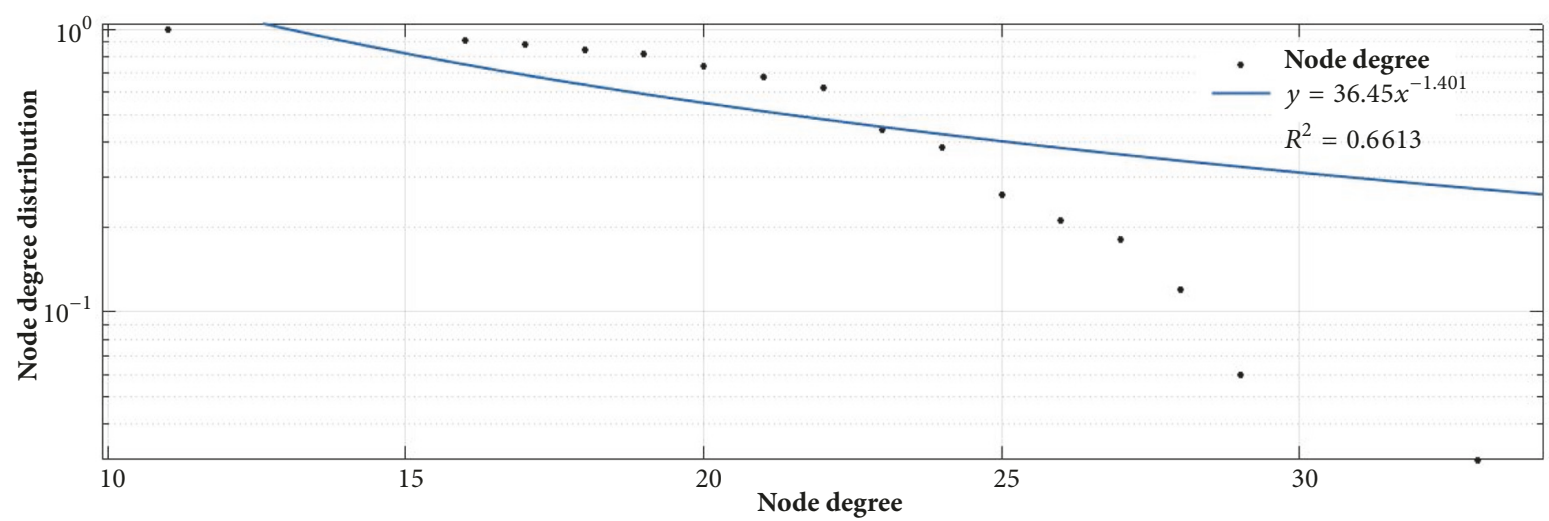

(a)

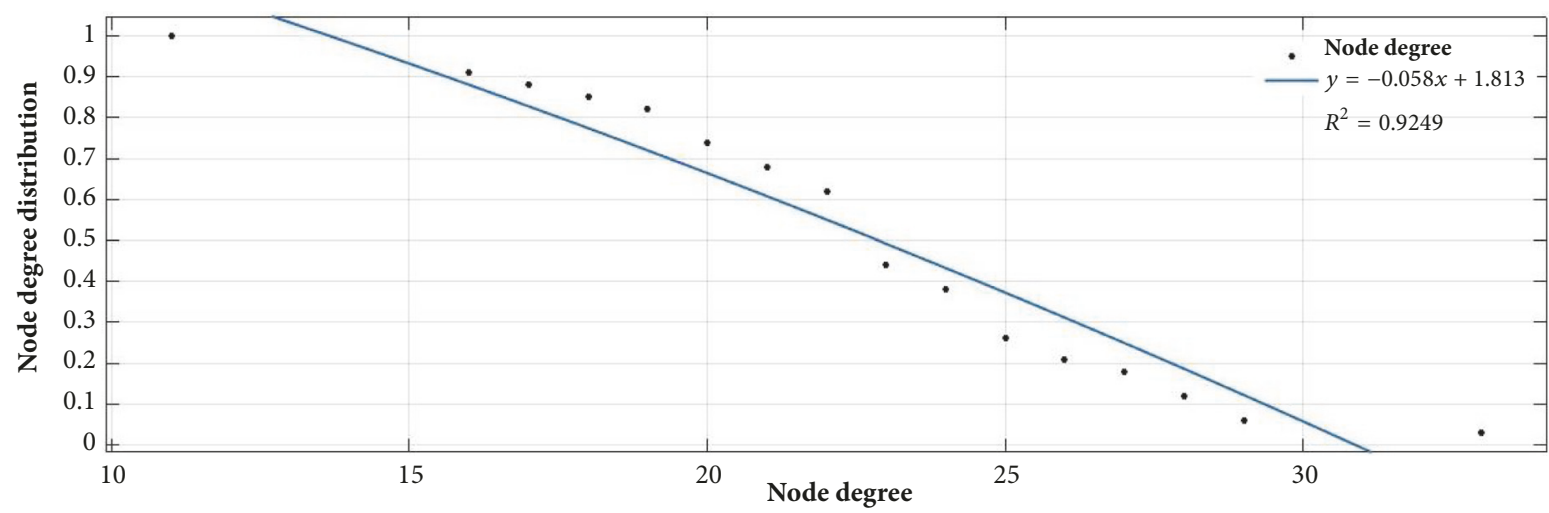

(b)

FIgURE 4: Node degree cumulative distribution: (a) obeying power law distribution; (b) obeying linear distribution.

$P(1.401)$ and $R^{2}=0.6613$ show that the regression effect is general and the cumulative distribution of node degree does not obey the power law distribution. In Figure 4(b), $R^{2}=0.9249$ shows that the cumulative distribution of node degrees obeys the linear distribution. Reference [36] pointed out that the cumulative distribution of nodal degrees in a scale-free network obeys the power law distribution. Since the cumulative distribution of the node degree in the regional traffic network does not obey the power law distribution, this traffic network does not belong to the scale-free network. In addition, [36] also proposed that the scale-free network has the ability to resist random attacks. This also indirectly shows that the urban regional road network does not have the same ability of the scale-free network resisting random attacks. In other words, the random attack such as a traffic accident can cause great damage to the traffic network, which further exemplifies the significance of this research.

\subsection{Feasibility Analysis of Cascading Failure Model}

3.2.1. Traffic Network Congestion Process in Simulation Environment. In order to analyze the feasibility of the cascade failure model proposed in this paper, VISSIM is used to build the simulation network. The simulation data also come from the Shenyang SCATS system. Part of the regional road network from Figure 3 is selected as the simulation object

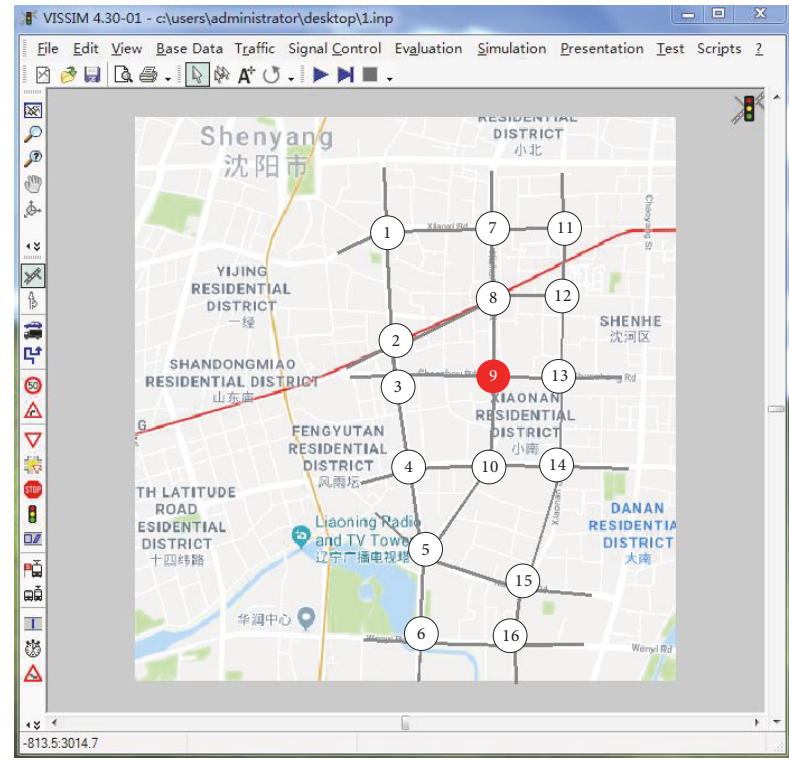

FIgURE 5: Simulation traffic network.

in Figure 5. The simulation network has 16 intersections and 44 roads (the opposite road counts as two roads). During the simulation process, assume that when a traffic accident 
TABLE 3: Statistics of new node degree in Shenyang regional traffic network.

\begin{tabular}{|c|c|c|c|c|c|c|c|c|c|c|c|c|c|c|c|c|}
\hline Node degree & 11 & 16 & 17 & 18 & 19 & 20 & 21 & 22 & 23 & 24 & 25 & 26 & 27 & 28 & 29 & 33 \\
\hline Node number & 3 & 1 & 1 & 1 & 3 & 2 & 2 & 6 & 2 & 4 & 2 & 1 & 2 & 2 & 1 & 1 \\
\hline
\end{tabular}

TABLE 4: Average travel time data before and after the accident.

\begin{tabular}{|c|c|c|c|c|c|c|c|c|c|c|c|c|c|c|}
\hline \multirow{2}{*}{ Road number } & \multicolumn{2}{|c|}{ Time step 0} & \multicolumn{2}{|c|}{ Time step 1} & \multicolumn{2}{|c|}{ Time step 2} & \multicolumn{2}{|c|}{ Time step 3} & \multicolumn{2}{|c|}{ Time step 4} & \multicolumn{2}{|c|}{ Time step 5} & \multicolumn{2}{|c|}{ Time step 6} \\
\hline & $\mathrm{AB}$ & $\mathrm{BA}$ & $\mathrm{AB}$ & BA & $\mathrm{AB}$ & BA & $\mathrm{AB}$ & $\mathrm{BA}$ & $\mathrm{AB}$ & BA & $\mathrm{AB}$ & BA & $\mathrm{AB}$ & BA \\
\hline $1-2$ & 49 & 53 & 50 & 55 & 54 & 53 & 58 & 59 & 58 & 77 & 60 & 125 & 61 & 133 \\
\hline $2-3$ & 10 & 12 & 11 & 13 & 13 & 15 & 12 & 25 & 13 & 28 & 13 & 50 & 13 & 61 \\
\hline $3-4$ & 41 & 40 & 40 & 50 & 43 & 64 & 49 & 94 & 50 & 89 & 50 & 111 & 51 & 134 \\
\hline $4-5$ & 36 & 42 & 40 & 39 & 44 & 43 & 43 & 57 & 42 & 74 & 42 & 91 & 45 & 95 \\
\hline $5-6$ & 34 & 39 & 35 & 40 & 37 & 35 & 40 & 51 & 40 & 53 & 40 & 53 & 42 & 56 \\
\hline $7-8$ & 33 & 29 & 33 & 34 & 44 & 35 & 47 & 43 & 68 & 71 & 75 & 77 & 78 & 80 \\
\hline $8-9$ & 30 & 31 & 44 & 47 & 61 & 48 & 115 & 80 & 117 & 107 & 123 & 134 & 166 & 175 \\
\hline $9-10$ & 44 & 40 & 51 & 53 & 83 & 86 & 120 & 106 & 141 & 133 & 153 & 149 & 177 & 169 \\
\hline $1-7$ & 50 & 52 & 49 & 50 & 51 & 48 & 49 & 51 & 53 & 51 & 50 & 53 & 53 & 52 \\
\hline $2-8$ & 50 & 54 & 51 & 50 & 53 & 51 & 64 & 54 & 73 & 67 & 114 & 103 & 145 & 120 \\
\hline $3-9$ & 48 & 47 & 55 & 59 & 81 & 88 & 100 & 111 & 108 & 146 & 152 & 159 & 186 & 194 \\
\hline $4-10$ & 40 & 41 & 40 & 37 & 39 & 41 & 50 & 53 & 54 & 53 & 88 & 86 & 91 & 112 \\
\hline $5-10$ & 55 & 50 & 51 & 56 & 58 & 55 & 73 & 71 & 75 & 74 & 77 & 76 & 77 & 76 \\
\hline 5-15 & 46 & 50 & 51 & 52 & 51 & 54 & 50 & 54 & 52 & 52 & 51 & 53 & 55 & 54 \\
\hline $6-16$ & 44 & 46 & 43 & 40 & 44 & 42 & 43 & 47 & 45 & 44 & 45 & 43 & 47 & 45 \\
\hline 7-11 & 33 & 35 & 31 & 30 & 31 & 32 & 37 & 32 & 35 & 34 & 37 & 35 & 37 & 37 \\
\hline $8-12$ & 31 & 35 & 33 & 34 & 34 & 34 & 36 & 45 & 46 & 48 & 67 & 71 & 90 & 79 \\
\hline $9-13$ & 30 & 29 & 39 & 44 & 61 & 66 & 74 & 78 & 76 & 119 & 121 & 130 & 179 & 201 \\
\hline $10-14$ & 40 & 37 & 36 & 34 & 33 & 35 & 40 & 51 & 53 & 77 & 52 & 85 & 81 & 109 \\
\hline $15-16$ & 25 & 23 & 26 & 27 & 23 & 23 & 25 & 23 & 22 & 24 & 25 & 25 & 27 & 26 \\
\hline $11-12$ & 34 & N/A & 32 & N/A & 31 & N/A & 46 & N/A & 74 & N/A & 75 & N/A & 100 & $\mathrm{~N} / \mathrm{A}$ \\
\hline $12-13$ & 40 & N/A & 39 & N/A & 46 & N/A & 76 & N/A & 93 & N/A & 110 & N/A & 180 & N/A \\
\hline $13-14$ & 47 & N/A & 48 & N/A & 46 & N/A & 63 & N/A & 92 & N/A & 101 & N/A & 106 & $\mathrm{~N} / \mathrm{A}$ \\
\hline $14-15$ & 66 & N/A & 65 & N/A & 59 & N/A & 69 & N/A & 85 & N/A & 92 & N/A & 92 & N/A \\
\hline
\end{tabular}

occurs at the intersection 9, the lanes of the entrance road are closed to simulate traffic accidents in the simulated environment.

After running the simulation program, the average travel time data of all the roads can be obtained in Table 4 .

In Table 4, $\mathrm{AB}$ and $\mathrm{BA}$ represent the direction of road. $\mathrm{N} / \mathrm{A}$ represents no roads of this direction. In time step 0 , the accident does not occur at the intersection 9. After time step 1 , the changes of the average travel time of all the roads under the accident are show in Table 4. According to the evaluation index system of the city traffic management, the traffic state of every road can be obtained after the traffic accident. The traffic state is divided into four levels, unblocked, slightly crowed, crowed and heavily crowed, which are expressed in four different colors. The spread of traffic congestion after the traffic accident in the traffic network is visually represented in Figure 6.

Figure 6 shows the evolution of the traffic congestion after the traffic accident. It can be seen that when the traffic accident occurs at the intersection 8 , the adjacent roads are not immediately crowded, but the traffic state transits from slightly crowed state to crowded state. Subsequently, due to the road capacity, the continuously increasing traffic volume is gradually saturated and the traffic states of other roads have gradually changed to crowded state. What is more, the roads around intersection 9 basically lose their transportation capacity, and a closed-loop failure network is formed in the simulation network.

\subsubsection{Traffic Network Failure Process under the Proposed} Cascading Failure Model. Based on the same actual data of Shenyang regional traffic network, the traffic network operation process after the random attack (i.e., the traffic accident at the intersection 8) is numerically simulated. According to the failure evaluation parameters of each road, the process of failure evolution can be analyzed and the path of failure propagation will be clarified in Table 5 .

The calculation formula of failed road ratio $\theta$ is as shown:

$$
\theta=\frac{\sum \theta_{i j}^{\prime}}{E}, \quad \theta_{i j}^{\prime}= \begin{cases}1, & e_{i j} \text { is failed } \\ 0, & \text { otherwise }\end{cases}
$$

where $E$ represents the number of edges $e_{i j}$ in the network. 


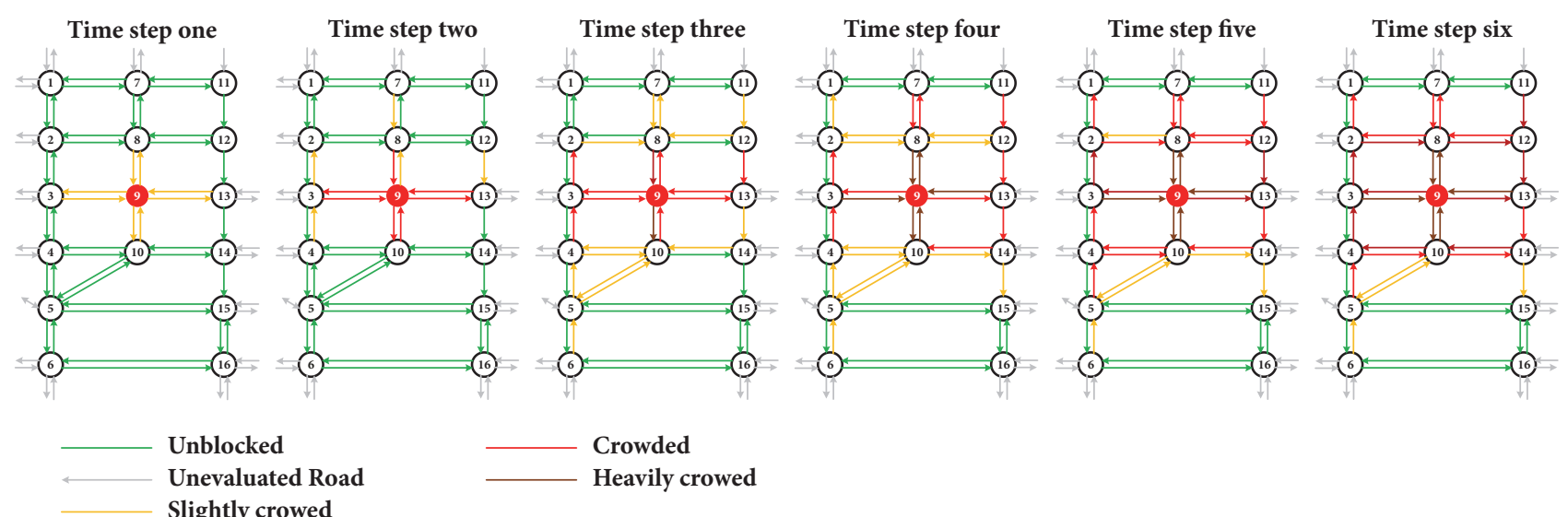

FIGURE 6: Schematic diagram of traffic congestion evolution.

TABLE 5: Cascade failure process of the simulation network.

\begin{tabular}{l}
\hline Cascade failure process \\
\hline$[(9,10)(10,9)] \longrightarrow[(8,9)(3,9)(13,9)] \longrightarrow[(9,3)(9,13)(9,8)] \longrightarrow[(3,2)(12,13)] \longrightarrow$ \\
{$[(4,3)(14,10)] \longrightarrow[(7,8)(8,7)(13,14)] \longrightarrow[(11,12)] \longrightarrow[(4,10)(10,4)(8,12)(12,8)] \longrightarrow$} \\
{$[(5,4)(2,8)] \longrightarrow[(8,2)(2,1)] \longrightarrow[(10,14)] *$} \\
$*$ " $\longrightarrow$ " represents the direction of the cascading failure propagation; "[ ]" represents the failed roads in the same time step; $(i, j)$ represents the road from \\
nodeito node $j$.
\end{tabular}

Compared with Figure 6, it is found that the failure propagation path in Table 5 is consistent with the traffic congestion propagation process, which indicates that the proposed cascade failure model can accurately reflect the failure process of the actual traffic network after encountering random attacks such as traffic accidents.

\subsection{Vulnerability Analysis of Urban Regional Traffic Network.} Combining the actual traffic flow data in Shenyang regional traffic network, Section 3.3 will grasp the vulnerable position of the urban traffic network. In the cascading failure simulation process, it is generally necessary to consider the changes of the traffic volume within the network, that is:

(1) The total traffic volume in the network remains unchanged: In the numerical simulation of the failure after the primary failure of the regional traffic network, no new traffic volume is generated in the regional traffic network.

(2) The total traffic volume in the network remains increased: During the failure numerical simulation after the primary failure in the regional traffic network, with considering that the new traffic demand is generated, the new traffic volume is input to the urban regional traffic network, which may cause the next round of network cascading failure.

To analyze the vulnerable ability of the traffic network, the first type of the above network traffic volume change modes; the total volume within the network remaining unchanged will be used. By setting the total traffic volume in the network to a fixed value, the failure law in the regional traffic network under random attacks can be discovered through a simulated failure process. In addition, if the total traffic volume in the network continues to increase, the traffic demand will be high and the vulnerable ability of the traffic network cannot be clearly reflected by the failure simulation results, because this simulation results are largely affected by the vulnerability of the traffic network and the external load. Therefore, the cascading failures under random attacks are simulated under the condition of the constant traffic volume of the regional traffic network.

3.3.1. Numerical Simulation Process. The numerical simulation process of the vulnerability analysis of the urban regional traffic network is shown in Figure 7. And, the numerical simulation of the cascading failure is as follows:

Step 1 (double-layer network model construction). Combined with the actual urban regional traffic network topology parameters and traffic flow information, the double-layer network model is established.

Step 2 (initialization). The corresponding parameters in the double-layer network model are initialized, and the initial traffic volume is allocated in the travel network.

Step 3 (generate new load). Based on the OPA model, the regional traffic network generates new traffic volume in $\lambda_{\ell}$ proportion (determined by actual traffic network data).

Step 4 (update the parameters). Recalculate the traffic volume at each intersection and road atktime in the regional travel network.

Step 5 (random attacks). If randomly attack a road, the road load rapidly rises beyond its maximum bearing capacity and then gets failed. The road status changes from "Normal" state 


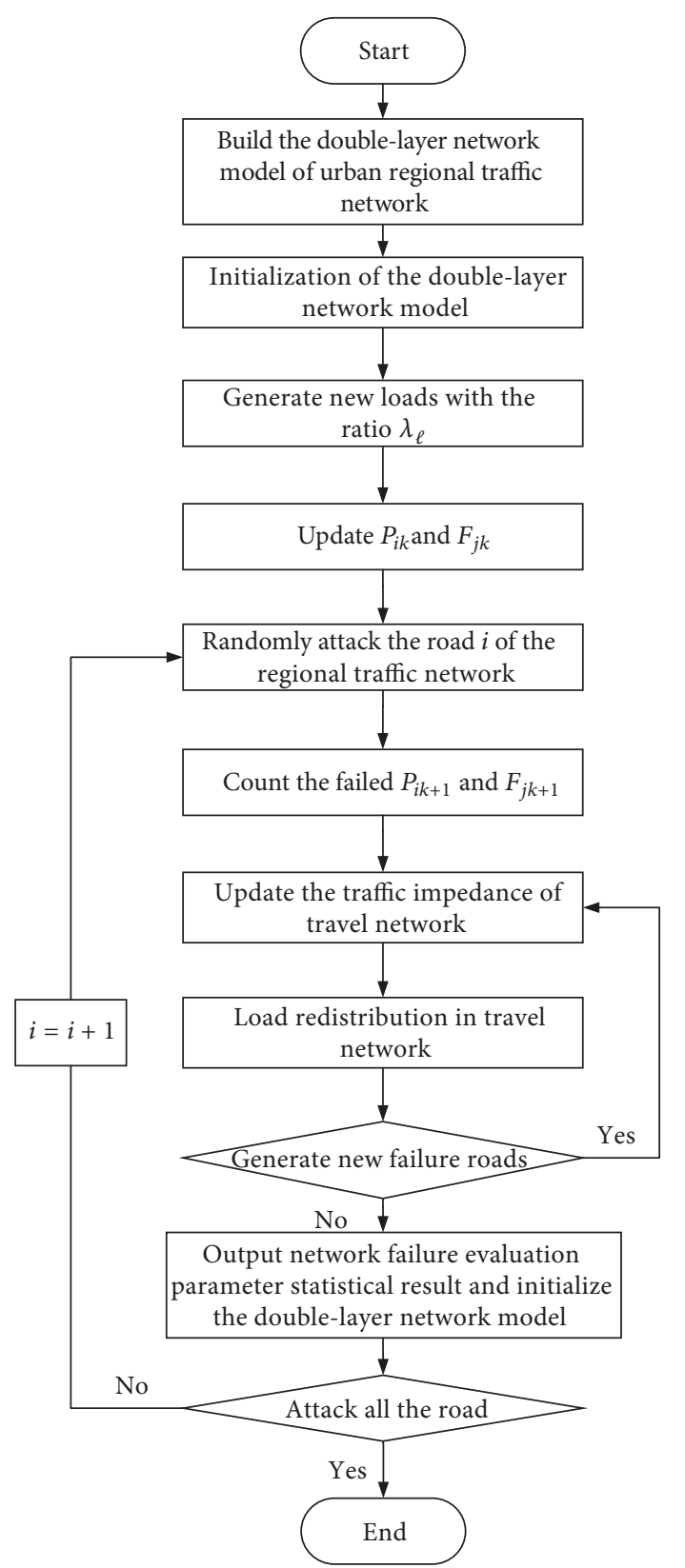

FIGURE 7: Numerical simulation process of failure model under random attacks.

to the "Transition" state, and finally go to "Failed" state. And, if attack intersection, the adjacent roads of this intersection will get failed and the traffic status will change to "Failed" state.

Step 6 (statistical parameters). The traffic volume $P_{i k+1}$ at each intersection $i$ and the traffic volume $F_{j k+1}$ on each road $j$ after the network failure at $k+1$ time.

Step 7 (impedance updating). When the state of the road changes to the "Failed" state, the impedance of the road will be adjusted to be infinite.

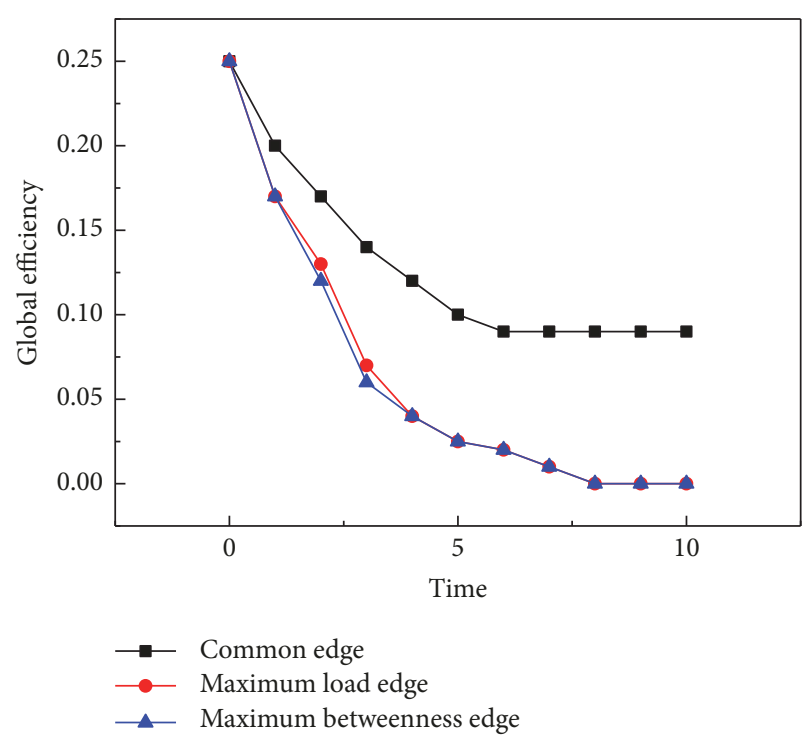

FIGURE 8: Influence of different attacked roads on the network global efficiency.

Step 8 (load redistribution). As the distribution of traffic impedance changes, the traffic volume distribution will change and be redistributed according to the principle of user equilibrium. The traveler can avoid the congested invalid road and choose a better travel path.

Step 9 (secondary failure identification). According to (12) and (13), clear the failed roads in the traffic network. If the secondary failure occurs, return to Step 7; otherwise, go to Step 10.

Step 10 (output result and initialize network). When no new failed road occurs, the statistical results of network evaluating parameters in the current failure are output and the traffic network model is restored to the initial configuration.

Step 11 (evaluation and judgment). When all the roads (or intersections) are not evaluated, skip to Step 5; otherwise, go to Step 12.

Step 12 (end). The failure results are counted, and end the numerical simulation.

\subsubsection{Numerical Simulation Result}

(1) Attacking the Roads. According to the simulation results, it is found that the edges with the largest load and the largest betweenness have the highest damage to the regional traffic network under random attacks in Figures 8 and 9. Among them, each simulation result is obtained after the simulation scheme independently performing 10 times.

From Figures 8 and 9, it can be seen that there are differences of the impact of the edge failure in the random attacks on the regional traffic network. And compared with the maximum load edge and the common edge, the degree of 


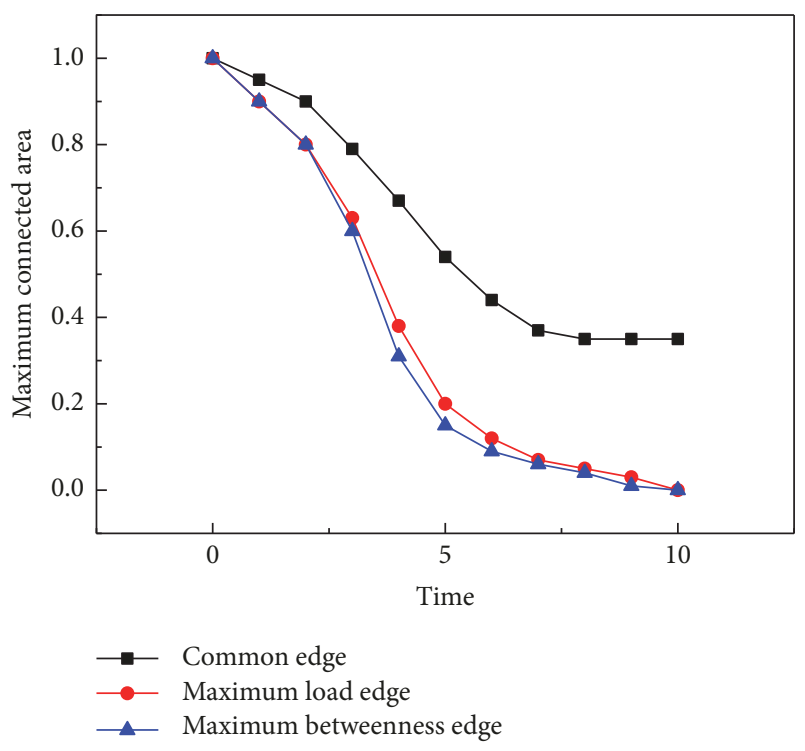

FIGURE 9: Influence of different attacked roads on the maximum connected area.

network failure caused by the maximum betweenness edge failed is the most serious.

In Figure 8, the reasons of the differences between three curves are as follows: After the vulnerable road attacked, the impedance of the failed road needs to be adjusted to be infinite. The traffic flow will be redistributed and the travel paths are adjusted accordingly. The global efficiency of the network is immediately affected by the failure. And, along with the failure propagation, the remaining road is relatively nonvulnerable roads in the late failure period. Nonvulnerable roads have little influence on the network topology and travel network. Therefore, in the late failure period, the network global efficiency tends to be stable.

Unlike Figure 8, in Figure 9, the three curves differ greatly. The same as in Figure 8 is that the damage caused by the largest betweenness edges attacked is the most. Whether the largest load edge or the largest betweenness edges in the urban regional traffic network is attacked, the loss of the maximum connected area is relatively small in the initial failure period. The reason is that after a fragile road failed, it will not immediately make the failure of the connected intersection. The failure condition of the intersection means that the adjacent roads are all failed. So, the loss of the maximum connected area in the initial failure period is reduced more slowly. With the continuous propagation of the failure, the adjacent roads and intersections get failed. As a result, in the mid-failure period, the loss of the maximum connected area drops sharply. Finally, in the late failure period, the loss of the maximum connected area also tends to be stable because the rest roads are mostly nonfragile.

Through the numerical simulation analysis of Figures 8 and 9, if traffic accidents randomly occur on the road with larger loads or larger betweenness, it is very easy to cause the network cascading failure. The roads with larger loads are mostly the trunk roads and bear the huge traffic volume. The

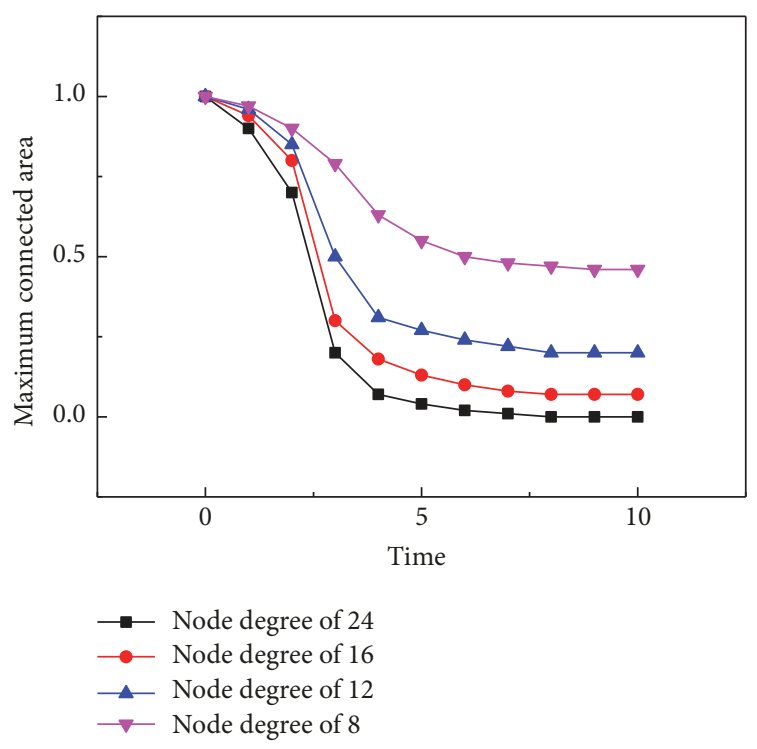

FIGURE 10: Influence of different node degrees on the maximum connected area.

larger betweenness roads are mostly the transport hubs and carry the transfer of the huge traffic volume. Therefore, the traffic management department needs to carry out a series of the traffic control measures such. At the same time, the traffic planning department is devoted to optimize the fragile sections of the traffic network.

(2) Attacking the Intersections. The size of intersections in the urban regional traffic network is different. Therefore, the common four-way intersections are taken as an example, which analyzes the influence of different node degrees under the cascading failure from the perspective of the maximum connected area.

There are many forms of the four-way intersections, such as the ones of the trunk roads, the ones between the trunk roads and the secondary trunk roads, the ones of the secondary trunk roads, the ones between the secondary trunk roads and the branch roads, and the ones of the branch roads. It is found that even if the intersection is in the form of the trunk roads, due to the different number of road lanes, the node degrees of these intersections are also different. By counting the node degrees of the four-way intersections in the traffic network in Figure 3, the five forms of intersections are respectively determined: (1) the intersections of the trunk roads (node degree is 24); (2) the intersections between the trunk roads and the secondary trunk roads (node degree is 20); (3) the intersections of the secondary trunk roads (node degree is 16); (4) the intersections between the secondary trunk roads and the branch roads (node degree is 12); (5) the intersections of the branch roads (node degree is 8). Based on the five node attack strategies, the maximum connected area is used to analyze the influence of the node degree on the network vulnerability.

In Figure 10, it can be seen that as the node degree increases, the failure degree of the traffic network gradually deepens. The reason is that the connection between nodes is 
closely related to the node degrees, namely, "the stronger the strong, the richer the rich". Therefore, when a node with high node degree gets attacked, there will be more nodes affected. Besides, the network failure speed will be higher, and the scope of the failed network will be also increased.

\section{Conclusions}

In order to solve the problem of identifying the vulnerable location of the regional traffic network under random attacks, this paper is divided into two parts to study: (1) to build the double-layer network model for clarifying the complexity of the regional traffic network; (2) to propose a cascading failure model of the regional traffic network under random attacks. Then through the numerical simulation results, the traffic network vulnerable sections will be determined.

First of all, in order to analyze the complex topological characteristics of urban traffic network, a double-layer network model is established. Among them, the lower layer is the regional road network model. The road network model refers to abstract the intersections into the nodes and the roads into the edges. And, the lower network model is an undirected complex network model. The upper layer is a regional travel network model. The model abstracts the OD points as the nodes and the travel paths as the edge. Therefore, it is a directed weighted complex network model.

Subsequently, the cascading failure model of the urban regional traffic network based on OPA is proposed. Based on the feasibility analysis, the proposed failure model is verified to accurately reflect the failure process. Finally, the numerical simulation is used to analyze the vulnerable ability of the regional traffic network.

\section{Data Availability}

The data used to support the findings of this study are included within the article.

\section{Conflicts of Interest}

The authors declare that they have no conflicts of interest.

\section{Acknowledgments}

This research is funded by the National Natural Science Foundation of China (no. 51308249).

\section{Supplementary Materials}

Part of the data used in this study. (Supplementary Materials)

\section{References}

[1] F. Wang X, X. Li, and R. G. Chen, Complex network theory and application, Tsinghua University Press, Beijing, China, 2006.

[2] J. Wu, Z. Y. Gao, and H. J. Sun, Urban traffic system complex, Beijing technology press, 2010.
[3] A. E. Motter and Y. C. Lai, "Cascade-based attacks on complex networks," Physical Review E Statistical Nonlinear \& Soft Matter Physics, vol. 66, no. 2, p. 065102, 2002.

[4] L. Zhao, K. Park, and Y. C. Lai, "Attack vulnerability of scalefree networks due to cascading breakdown," Physical Review E Statistical Nonlinear \& Soft Matter Physics, vol. 70 (3 Pt 2), p. 035101, 2004.

[5] P. Crucitti, V. Latora, and M. Marchiori, "Model for cascading failures in complex networks," Physical Review E: Statistical, Nonlinear, and Soft Matter Physics, vol. 69, no. 4, p. 045104, 2004.

[6] R. Kinney, P. Crucitti, R. Albert, and V. Latora, "Modeling cascading failures in the North American power grid," The European Physical Journal B, vol. 46, no. 1, pp. 101-107, 2005.

[7] D. J. Watts, "A simple model of global cascades on random networks," Proceedings of the National Acadamy of Sciences of the United States of America, vol. 99, no. 9, pp. 5766-5771, 2002.

[8] C. Asavathiratham, The influence model: A tractable representation for the dynamics of networked markov chains, Massachusetts Institute of Technology, 2001.

[9] I. Dobson, J. Chen, J. S. Thorp, B. A. Carreras, and D. E. Newman, "Examining criticality of blackouts in power system models with cascading events," in Proceedings of the 35th Annual Hawaii International Conference on System Sciences (HICSS '02), January 2002.

[10] I. Dobson, B. Carreras, and D. Newman, "A probabilistic loading-dependent model of cascading failure and possible implications for blackouts," in Proceedings of the 36th Annual Hawaii International Conference on System Sciences, 2003. Proceedings of the, p. 10 pp., Big Island, HI, USA, January 2003.

[11] X. F. Wang and J. Xu, "Cascading failures in coupled map lattices," Physical Review E: Statistical, Nonlinear, and Soft Matter Physics, vol. 70, no. 2, p. 056113, 2004.

[12] L. Buzna, K. Peters, and D. Helbing, "Modelling the dynamics of disaster spreading in networks," Physica A: Statistical Mechanics and its Applications, vol. 363, no. 1, pp. 132-140, 2006.

[13] G. W. Wen, J. N. Shun, Y. Y. Hong, and C. F. Wei, "Modeling the dynamics of disaster spreading from key nodes in complex networks," International Journal of Modern Physics C, vol. 18, no. 5, pp. 889-901, 2007.

[14] J. J. Wu, Z. Y. Gao, and H. J. Sun, "Effects of the cascading failures on scale-free traffic networks," Physica A Statistical Mechanics \& Its Applications, vol. 378, no. 2, pp. 505-511, 2007.

[15] J. Wu, H. Sun J, and Y. Gao Z, "Cascading failures on weighted urban traffic equilibrium networks," Physica A Statistical Mechanics \& Its Applications, vol. 386, no. 1, pp. 407-413, 2007.

[16] J. F. Zheng, Z. Y. Gao, and X. M. Zhao, "Modeling cascading failures in congested complex networks," Physica A Statistical Mechanics \& Its Applications, vol. 385, no. 2, pp. 700-706, 2007.

[17] J. J. Wu, Studies on the Complexity of Topology Structure in the Urban Traffic Network, Beijing Jiaotong University, 2008.

[18] Z. W. Wang, S. Peng, and Z. X. Huang, "Disaster sprawling dynamics model for the cascading failure in urban road traffic network," Journal of Safety and Environment, vol. 14, no. 3, 2014.

[19] S. Peng, Disaster sprawling dynamics model for the cascading failure in urban road traffic network, Changsha University of Science \& Technology, Changsha, China, 2014.

[20] Z. Cheng, J. Cao, and T. Hayat, "Cascade of failures in interdependent networks with different average degree," International Journal of Modern Physics C, vol. 25, no. 5, 2014. 
[21] Z. Huang, C. Wang, M. Stojmenovic, and A. Nayak, "Characterization of cascading failures in interdependent cyber-physical systems," IEEE Transactions on Computers, vol. 64, no. 8, pp. 2158-2168, 2015.

[22] S. Li, L. Li, Y. Yang, and Q. Luo, "Revealing the process of edgebased-attack cascading failures," Nonlinear Dynamics, vol. 69, no. 3, pp. 837-845, 2012.

[23] S. Chattopadhyay, H. Dai, D. Y. Eun, and S. Hosseinalipour, "Designing Optimal Interlink Patterns to Maximize Robustness of Interdependent Networks Against Cascading Failures," IEEE Transactions on Communications, vol. 65, no. 9, pp. 3847-3862, 2017.

[24] S. Ruj and A. Pal, "Analyzing cascading failures in smart grids under random and targeted attacks," in Proceedings of the 28th IEEE International Conference on Advanced Information Networking and Applications, IEEE AINA 2014, pp. 226-233, Canada, May 2014.

[25] A. Shen, J. Guo, and Z. Wang, "Analysis of Cascading Failures of Interdependent Networks under Random Attacks," DEStech Transactions on Computer Science and Engineering, no. wcne, 2016.

[26] S. Porta, P. Crucitti, and V. Latora, "The network analysis of urban streets: a primal approach," Environment \&amp; Planning B Planning \& Design, vol. 33, no. 2, pp. 853-866, 2006.

[27] Q. L. Liu, Research on complex networks reliability, Beijing University of Posts And Telecommunications, Beijing, China, 2007.

[28] R. Cohen, K. Erez, D. Ben-Avraham, and S. Havlin, "Breakdown of the internet under intentional attack," Physical Review Letters, vol. 86, no. 16, pp. 3682-3685, 2001.

[29] R. Albert and A. Barabási, "Statistical mechanics of complex networks," Reviews of Modern Physics, vol. 74, no. 1, pp. 47-97, 2002.

[30] P. Holme, B. J. Kim, C. N. Yoon, and S. K. Han, "Attack vulnerability of complex networks," Physical Review E: Statistical, Nonlinear, and Soft Matter Physics, vol. 65, no. 5, part 2, p. 056109, 2002.

[31] J. W. Wang, Study on cascading failure models on networks, Dalian University of Technology, Dalian, China, 2010.

[32] C. Q. Liu, Modern traffic planning, People's Transportation Press, Beijing, China, 2001.

[33] S. Z. Guo, Complex network basic theory, China Science Publishing \& Media Lid. (CSPM), Beijing, China, 2012.

[34] J. J. Wu, Z. Y. Gao, and H. J. Sun, "Model for dynamic traffic congestion in scale-free networks," EPL (Europhysics Letters), vol. 76, no. 5, pp. 787-793, 2006.

[35] D. J. Watts and S. H. Strogatz, "Collective dynamics of 'smallworld' networks," Nature, vol. 393, no. 6684, p. 440, 1998.

[36] R. Albert, H. Jeong, and A.-L. Barabási, "Error and attack tolerance of complex networks," Nature, vol. 406, no. 6794, pp. 378-382, 2000. 


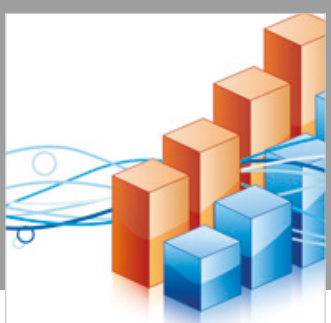

Advances in

Operations Research

\section{-n-m}
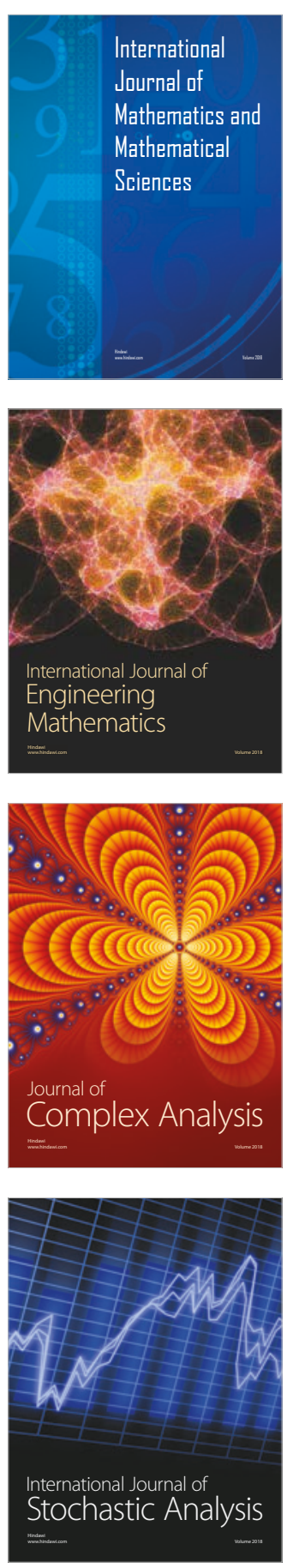
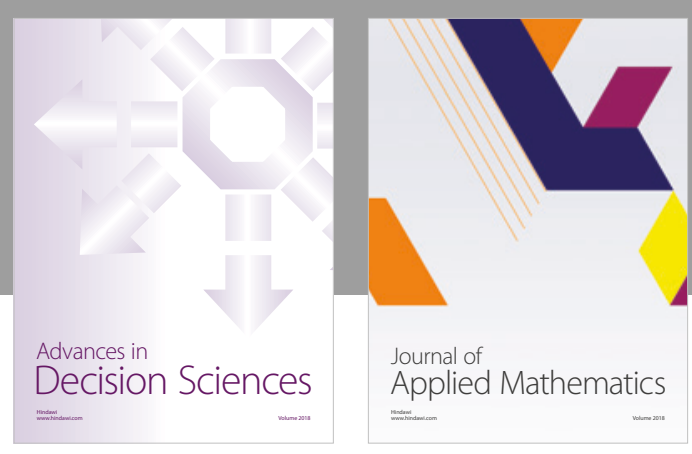

Journal of

Applied Mathematics
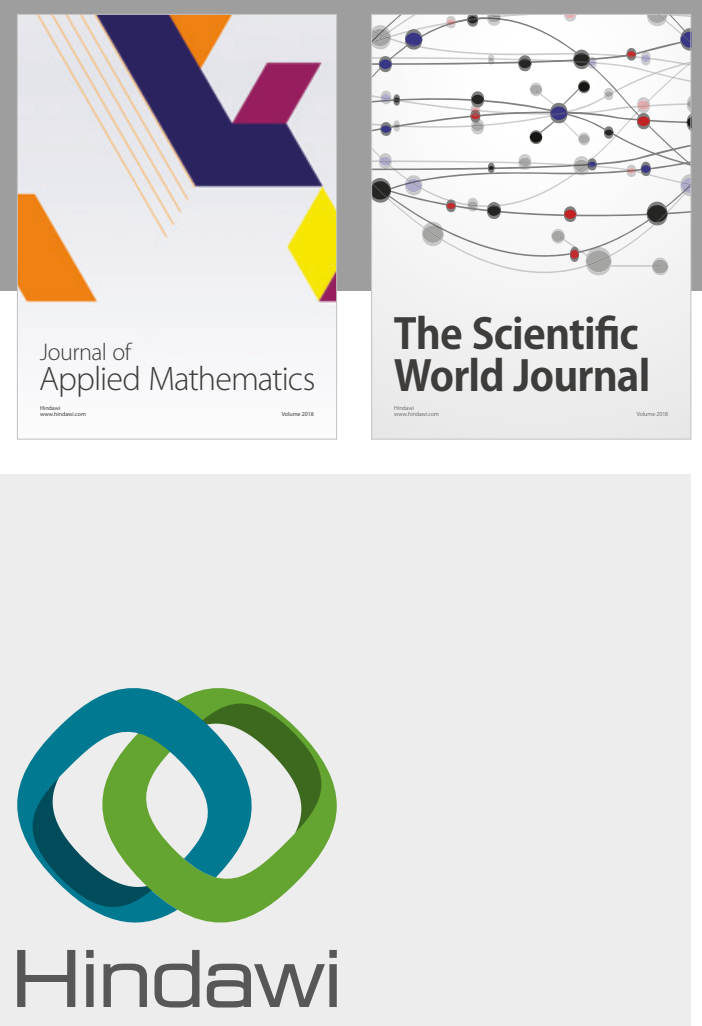

Submit your manuscripts at

www.hindawi.com

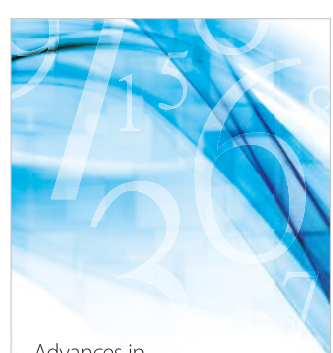

Advances in
Numerical Analysis
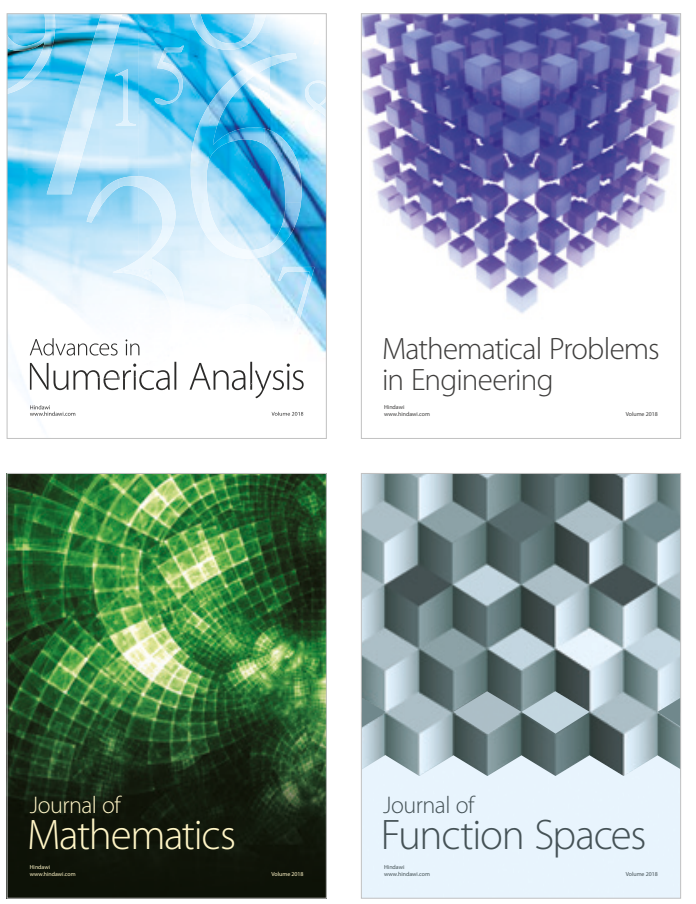

Mathematical Problems in Engineering

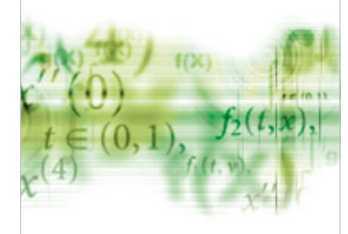

International Journal of

Differential Equations

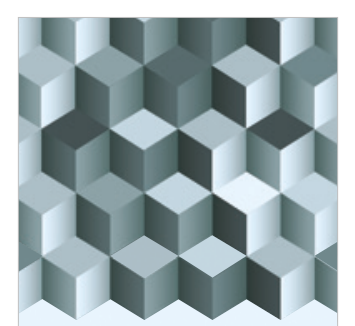

Journal of

Function Spaces

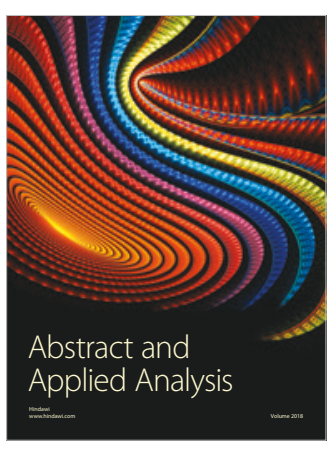

The Scientific

World Journal

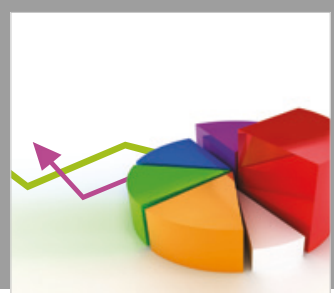

Journal of

Probability and Statistics
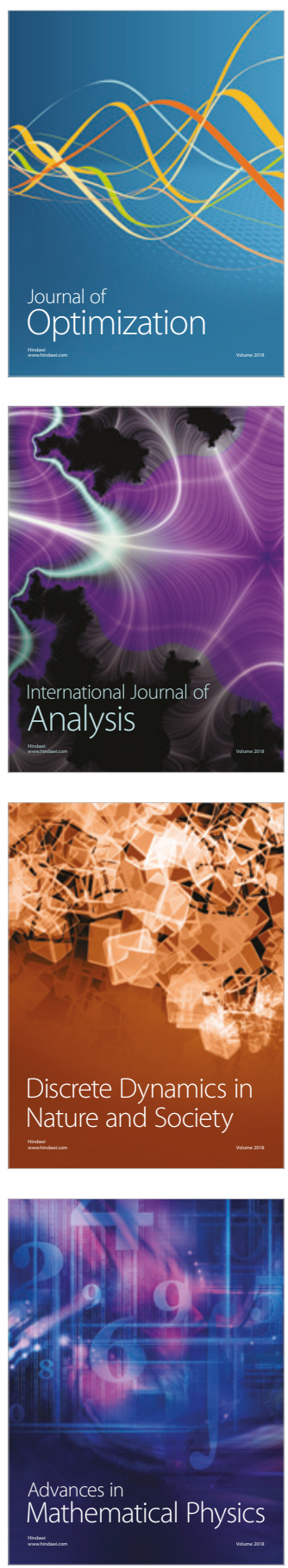